\title{
Charge Carrier Dynamics and Mobility Determined by Time-Resolved Terahertz Spectroscopy on Films of Nano-to-Micrometer-Sized Colloidal Tin(II) Monosulfide
}

Brian G. Alberding, ${ }^{1}$ Adam J. Biacchi, ${ }^{2}$ Angela R. Hight Walker, ${ }^{2}$ and Edwin J. Heilweil ${ }^{*}, 1$

${ }^{1}$ Radiation Physics Division and ${ }^{2}$ Engineering Physics Division, National Institute of Standards and Technology (NIST), 100 Bureau Drive, Gaithersburg, Maryland, 20899, United States

\section{Supporting Information}

\section{Figure S1}
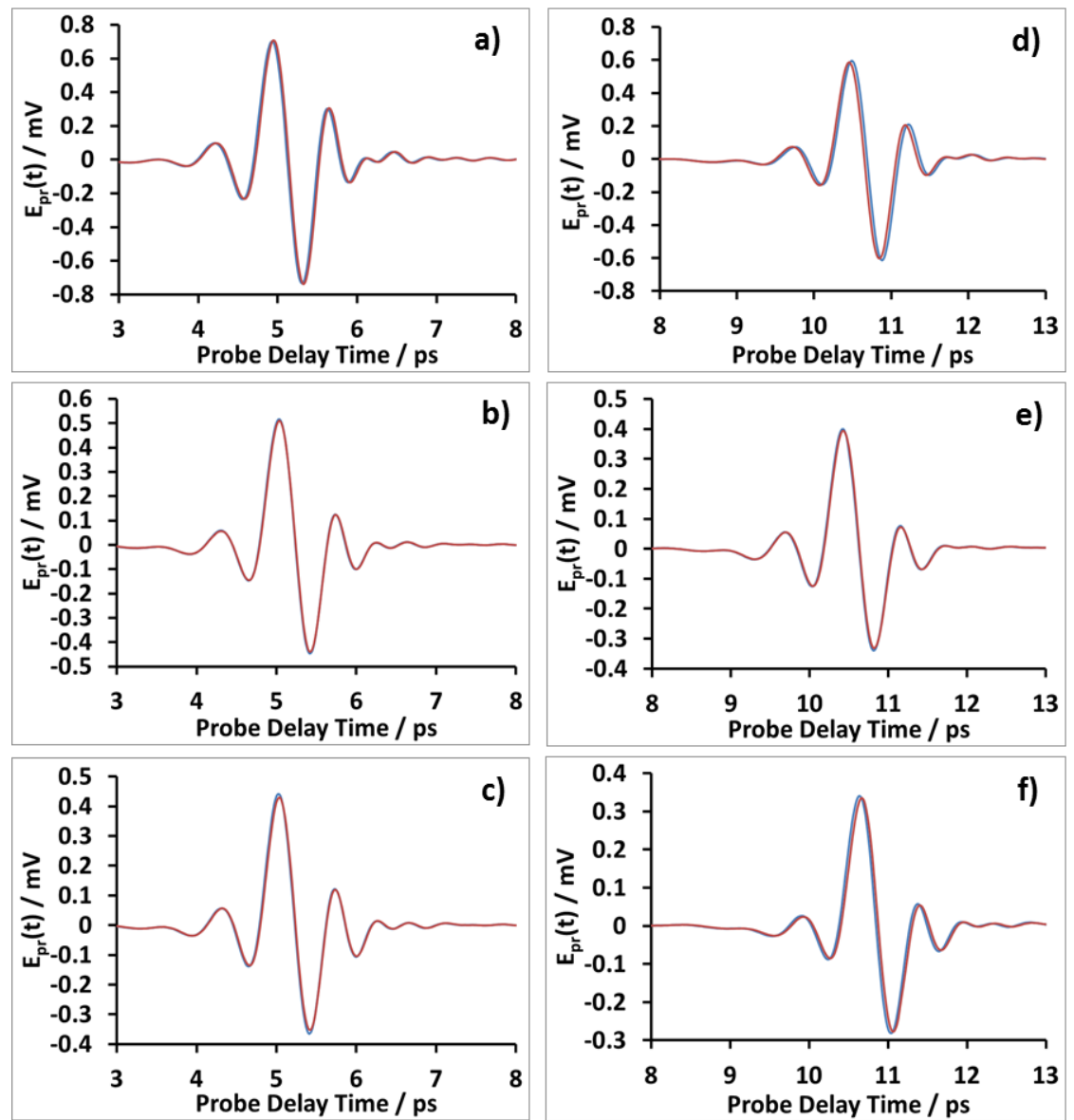

Figure S1: Measured transmission of the terahertz electric field, $E_{0}\left(t_{p r}\right)$ through the sample (red) or blank substrate (blue) as a function of the delay time between the probe and the gate in purged air at room temperature. The SnS dropcast samples are a) large b) medium c) small and for the SnS annealed samples d) large e) medium f) small. The signals are the raw transmission without any normalization. 


\section{Figure S2}
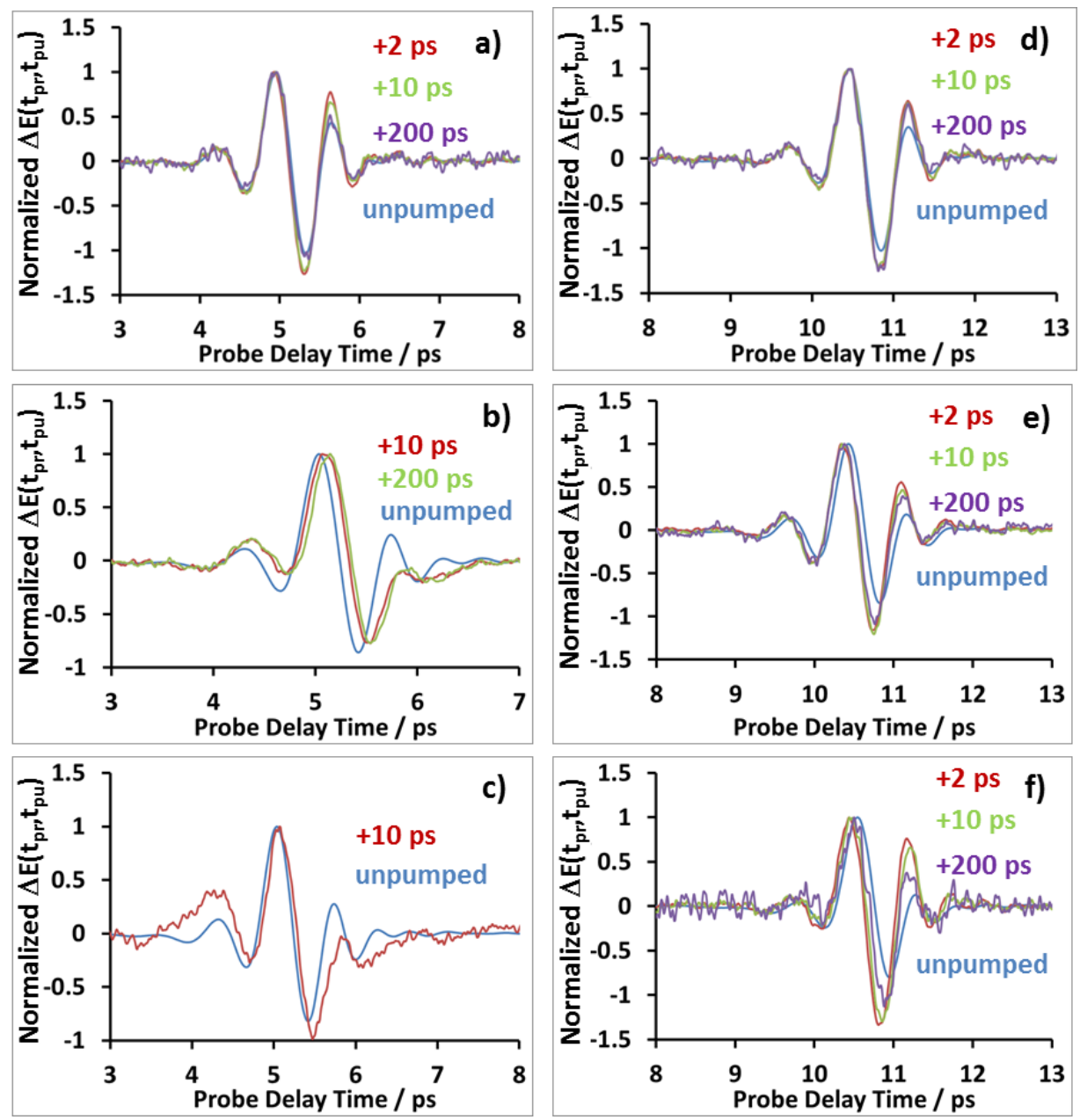

Figure S2: Normalized differential transmission of the terahertz electric field $\Delta \mathrm{E}\left(\mathrm{t}_{\mathrm{pr},}, \mathrm{t}_{\mathrm{pu}}\right)$ following photoexcitation at the indicated pump delay times compared to the transmission of the terahertz electric field, $E_{0}\left(t_{p r}\right)$, of the unpumped sample in purged air at room temperature. The changes $\Delta \mathrm{E}\left(\mathrm{t}_{\mathrm{pr}}, \mathrm{t}_{\mathrm{pu}}\right)$ are less than $2 \%$ of the unpumped, $\mathrm{E}_{0}\left(\mathrm{t}_{\mathrm{pr}}\right)$ signal. The SnS dropcast samples are a) large b) medium c) small and the SnS annealed samples are d) large e) medium f) small. The true sign of the differential transmission is negative compared to the unpumped sample indicating that the transmission decreases upon photoexcitation. 


\section{Figure S3}
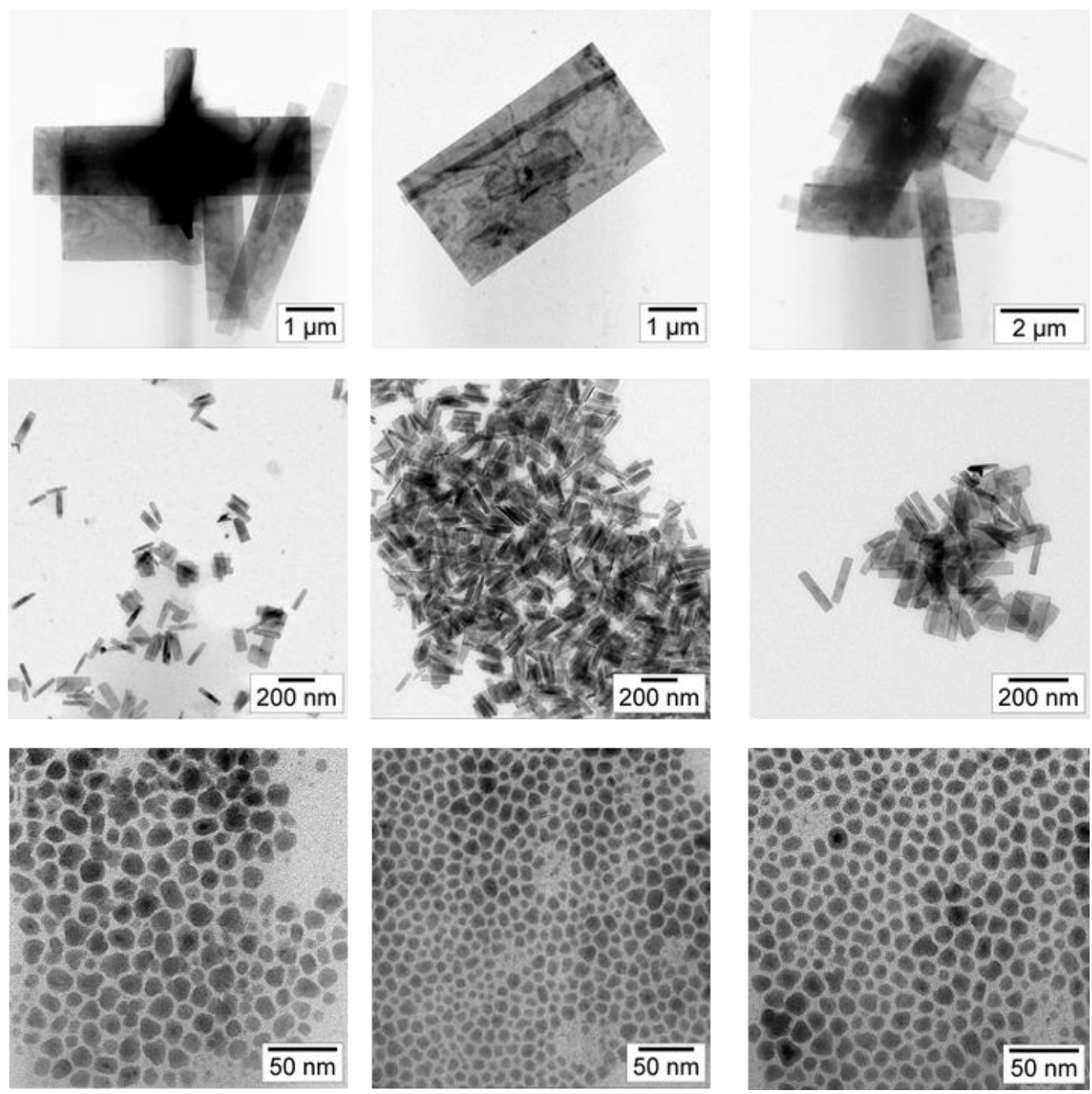

Figure S3: Additional TEM images of the SnS large (top), SnS medium (middle), and SnS small (bottom) samples. 


\section{Figure S4}
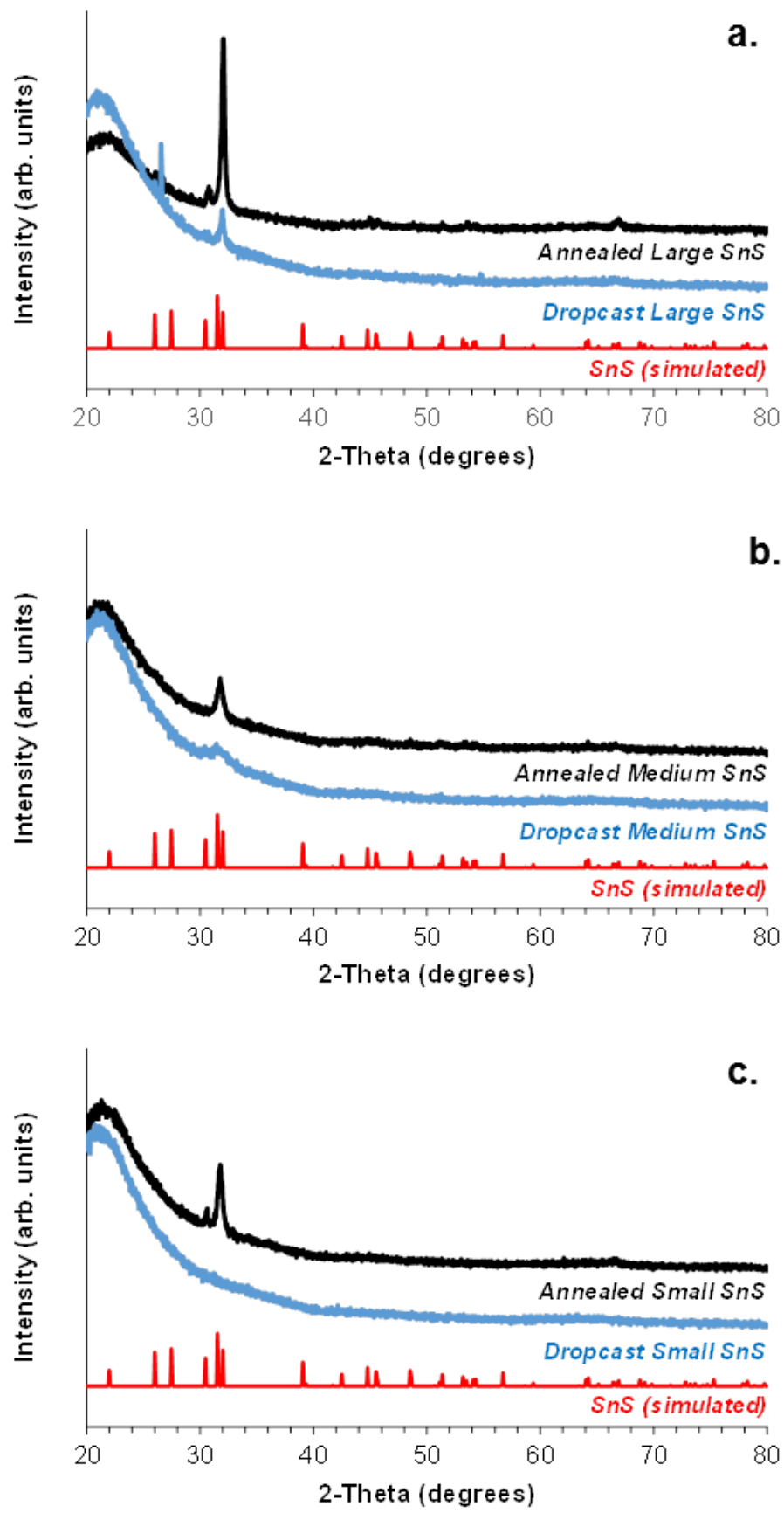

Figure S4: X-ray diffraction patterns for the dropcast (blue) and annealed (black) films of the (a) SnS Large, (b) SnS medium, and (c) SnS small samples that were used for the optical absorption and TRTS measurements. Note that the low signal intensity observed in these measurements is due to the fact that they were collected on fused quartz substrates instead of a zero background plate. 


\section{Figure S5}

a)

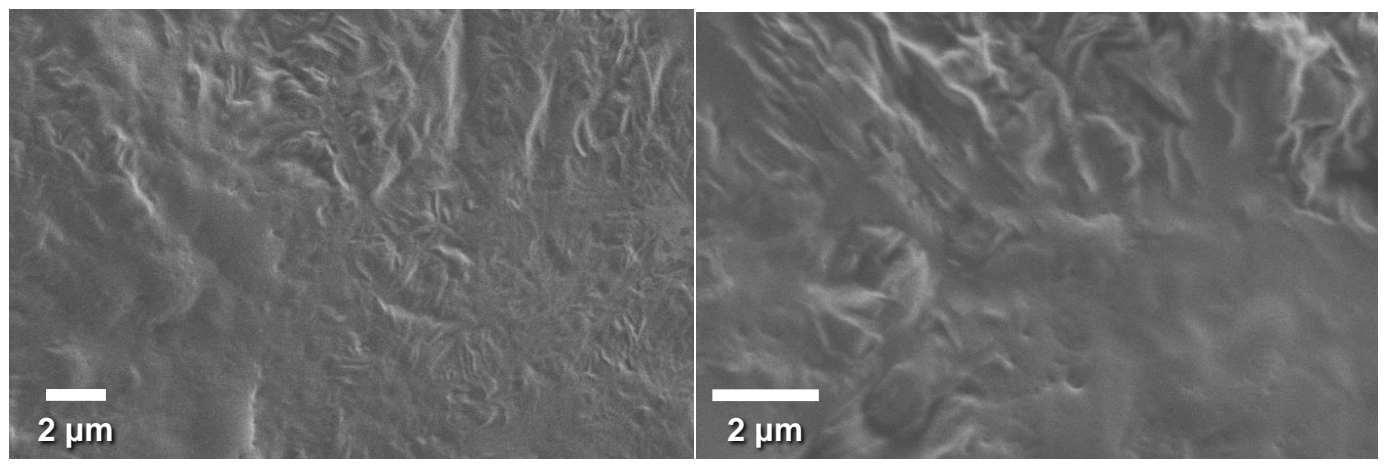

b)

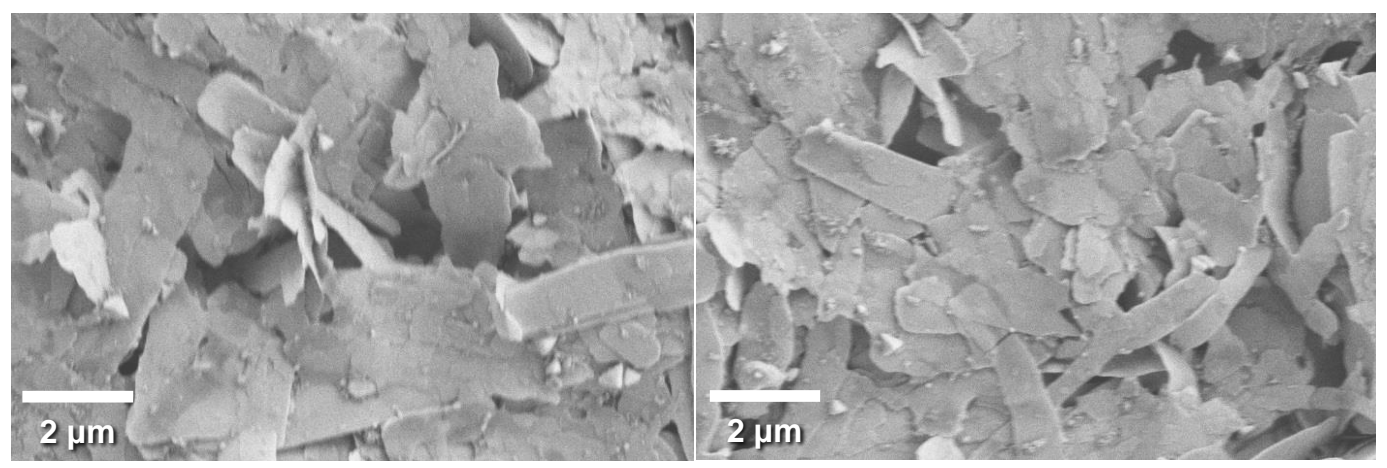

c)
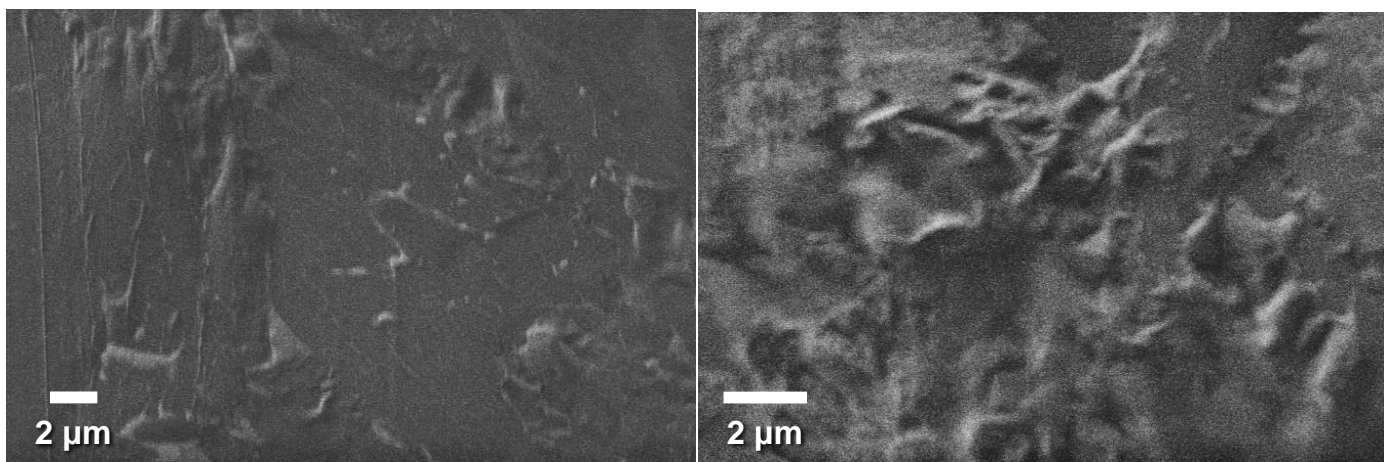
d)
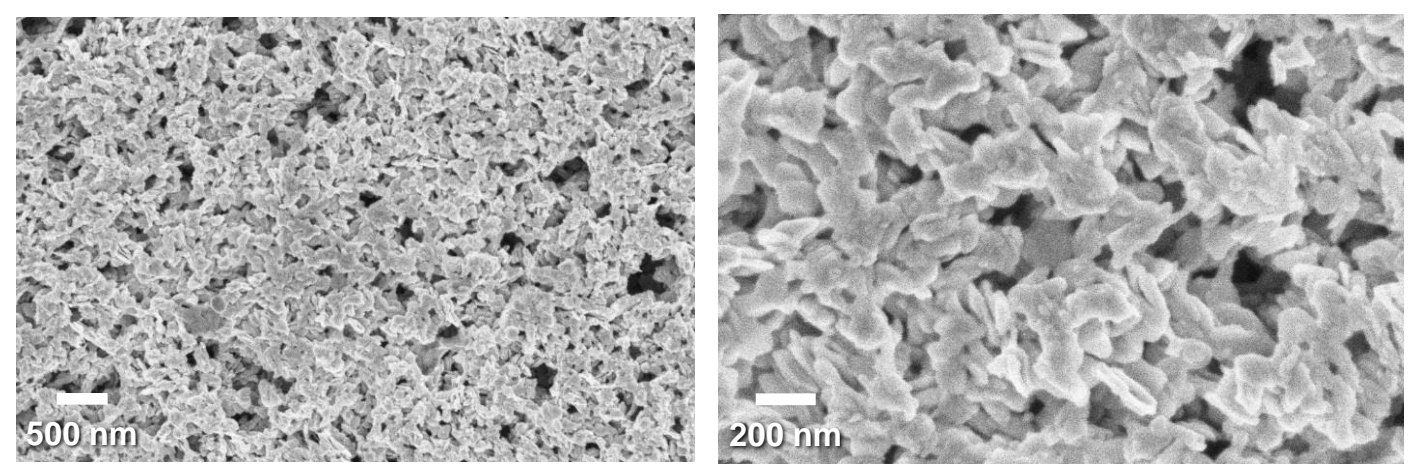

e)

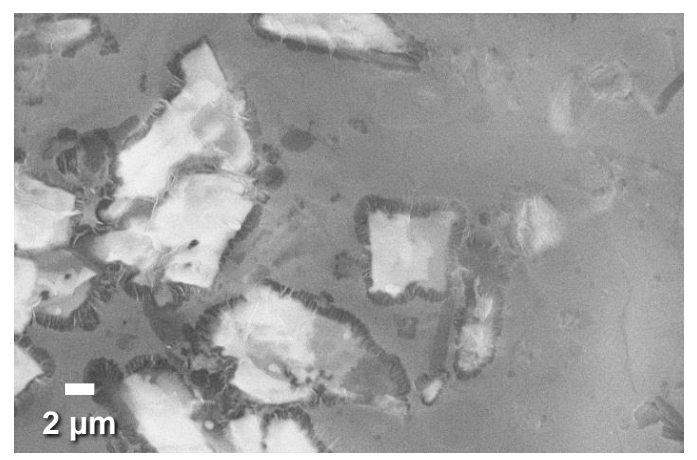

f)
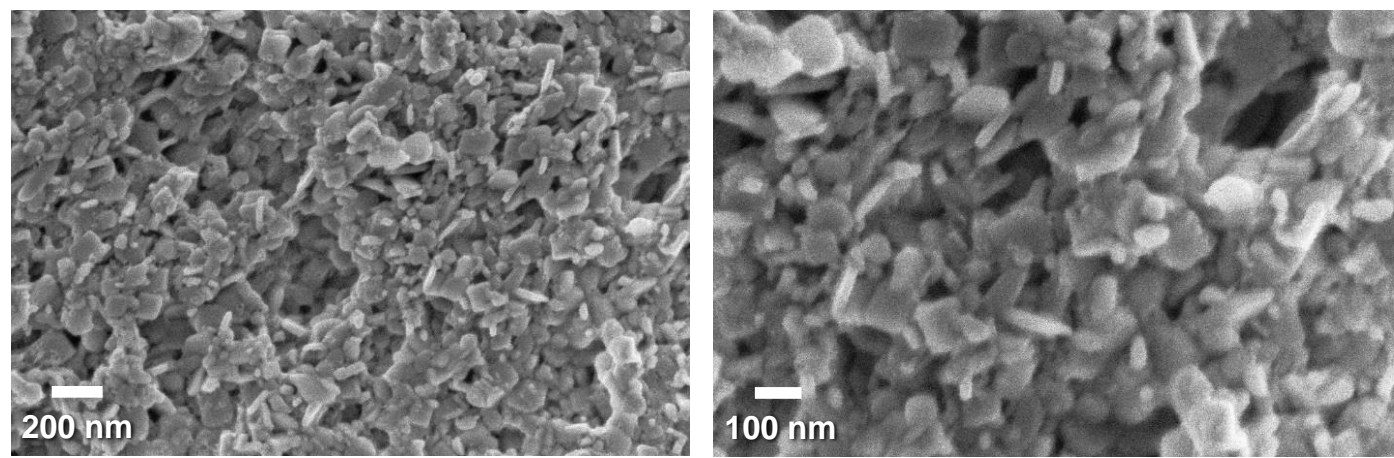

Figure S5: SEM images of analyzed SnS colloidal films, a) unannealed and b) annealed SnS large nanosheets, c) unannealed and d) annealed SnS medium nanosheets, e) unannealed and f) annealed SnS small nanoparticles. Note that there are some voids in the film of unannealed SnS small. Thickness approximations of individual annealed 2D crystals are SnS large, $30 \mathrm{~nm}$; SnS medium and small, $25 \mathrm{~nm}$. 


\section{Figure S6}
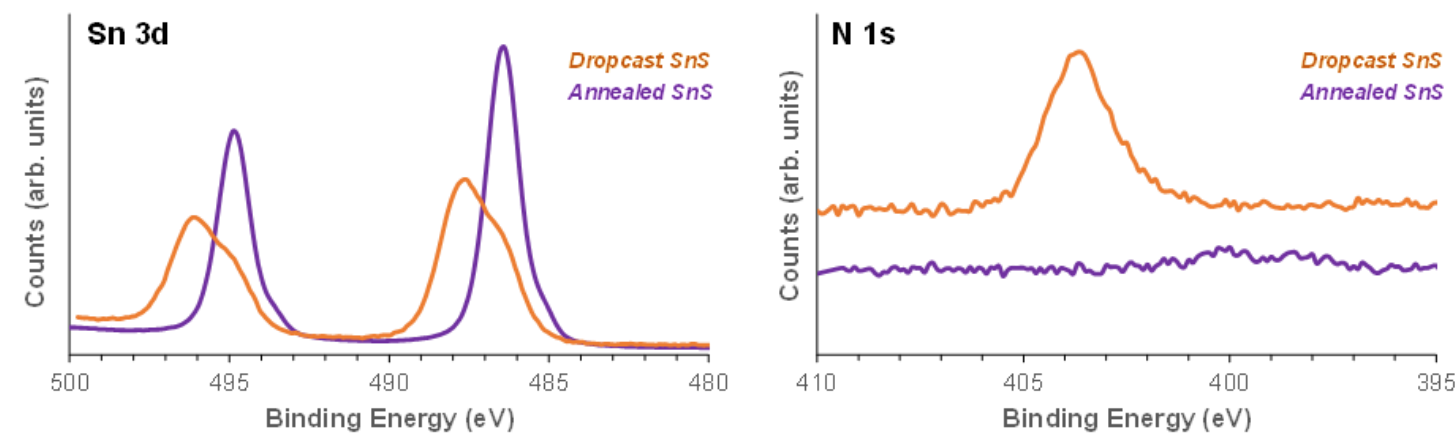

Figure S6: XPS spectra of the SnS medium dropcast (orange) and annealed (purple) samples in the Sn 3d (left) and N $1 \mathrm{~s}$ (right) regions.

\section{Figure S7}
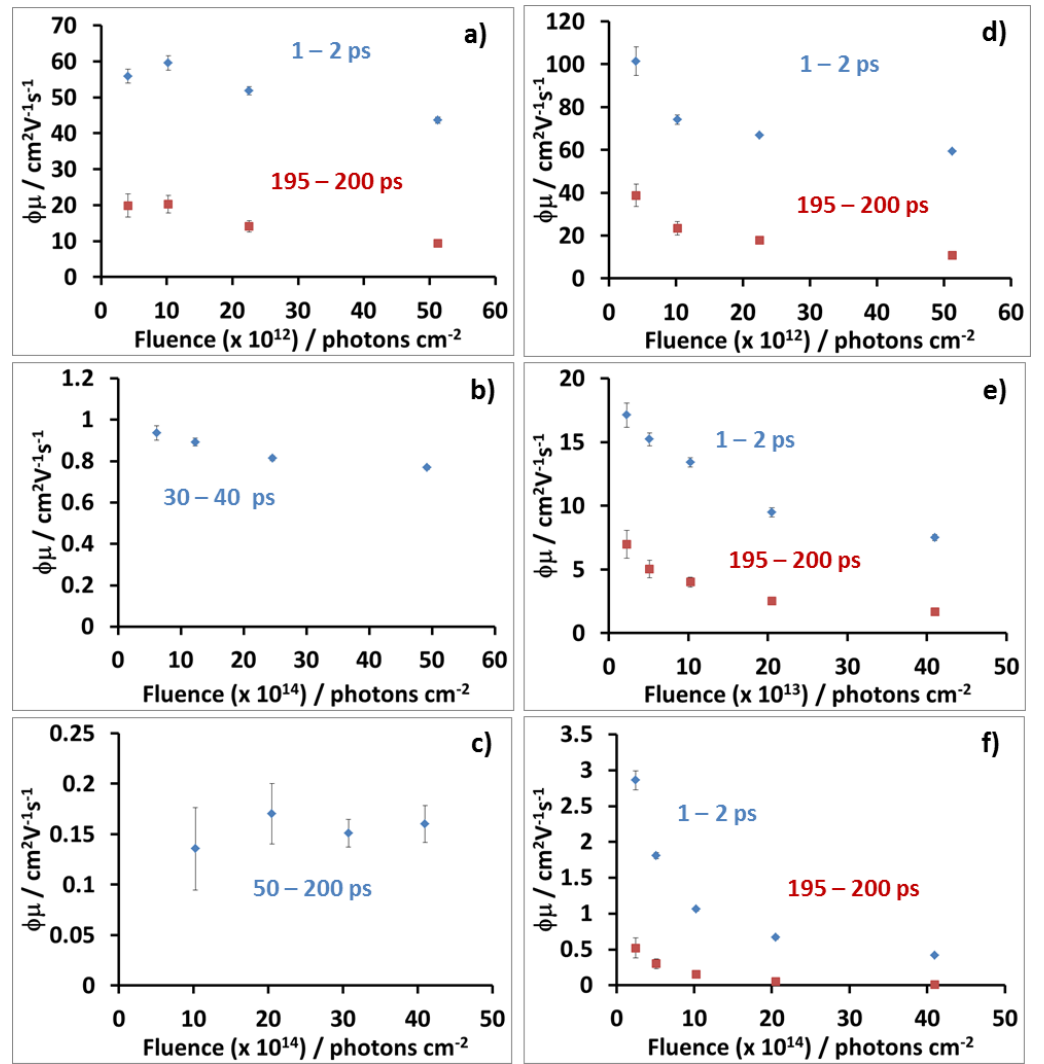

Figure S7: Fluence dependence of the mobilities calculated for each sample by taking an average of $\Delta \mathrm{E}\left(\mathrm{t}_{\text {pu }}\right) / \mathrm{E}_{0}\left(\mathrm{t}_{\mathrm{pr}}\right)$ across the indicated pump-probe time delays. The error bars represent the standard deviation of the average. The SnS dropcast samples are a) large b) medium c) small and the SnS annealed samples are d) large e) medium f) small. 


\section{Figure S8}
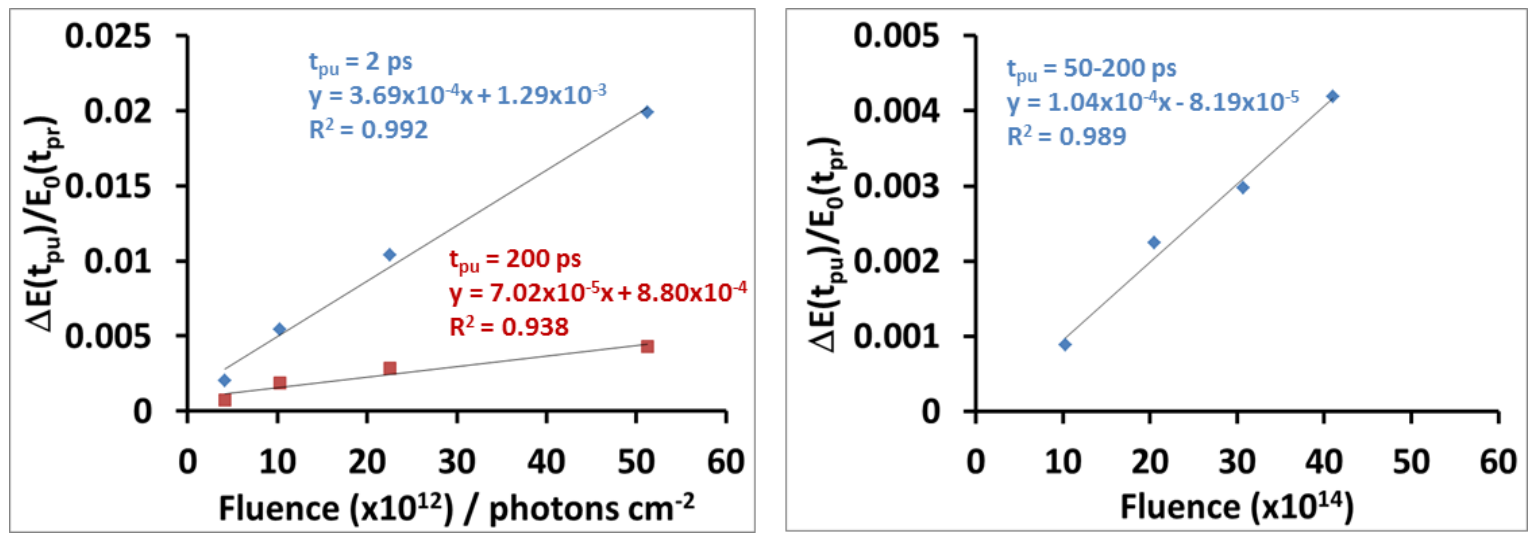

Figure S8: Representative fluence dependence of the measured change in terahertz electric field transmission following photoexcitation at $800 \mathrm{~nm}$ for the SnS large dropcast sample (left) and the SnS small dropcast sample (right) averaged over the indicated pump time delays. 


\section{Figure S9}
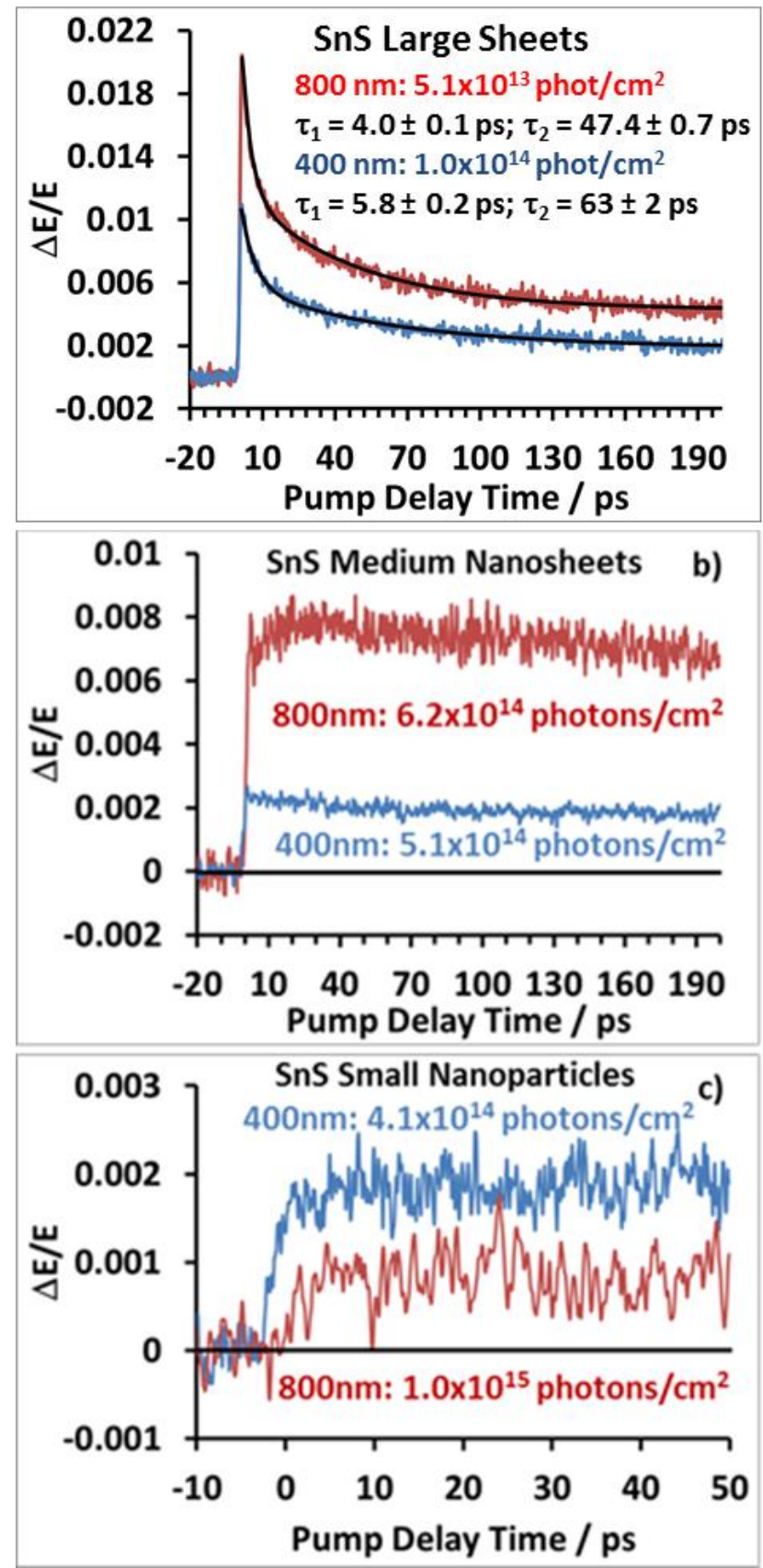

Figure S9: A comparison of the pump scan decay dynamics observed following $400 \mathrm{~nm}$ and $800 \mathrm{~nm}$ excitation at the indicated fluence for (a)the SnS large sheets, (b) the SnS medium nanosheets, and (c) the SnS small nanoparticles. 
Figure S10
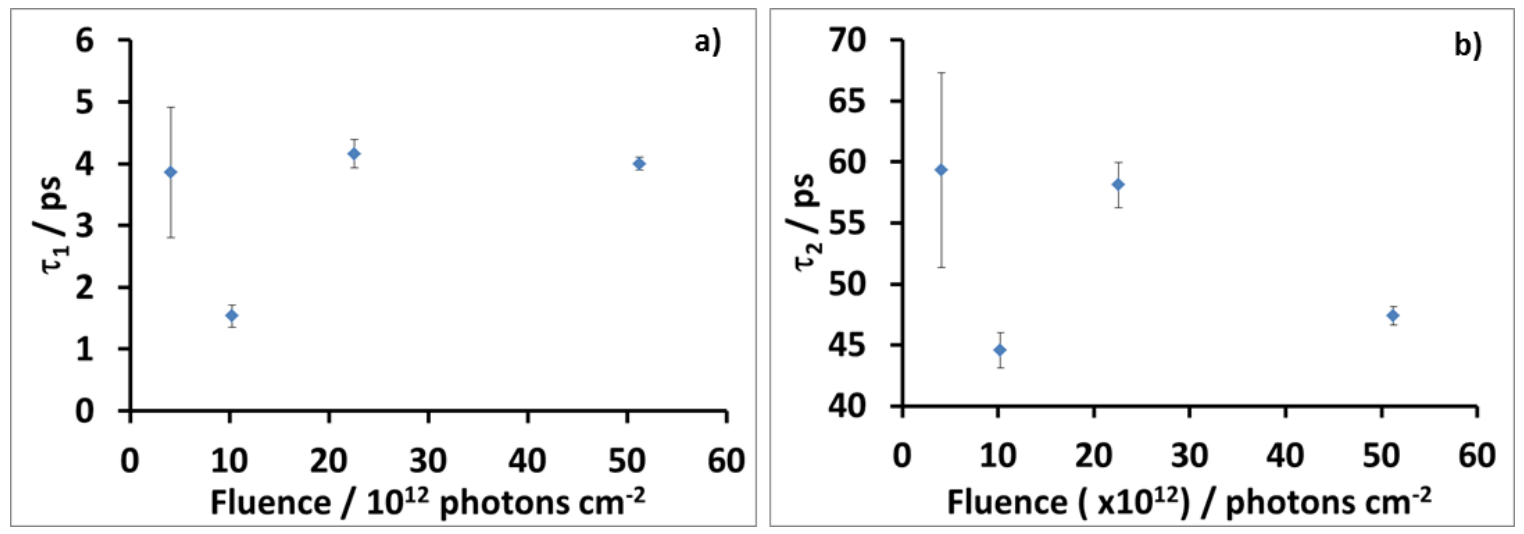

Figure S10: Fluence dependence of the first $\left(\tau_{1}\right.$, left) and second $\left(\tau_{2}\right.$, right) decay lifetime components from the fits of the pump-probe delay dynamics following photoexcitation at $800 \mathrm{~nm}$ for the SnS large film.

\section{Figure S11}

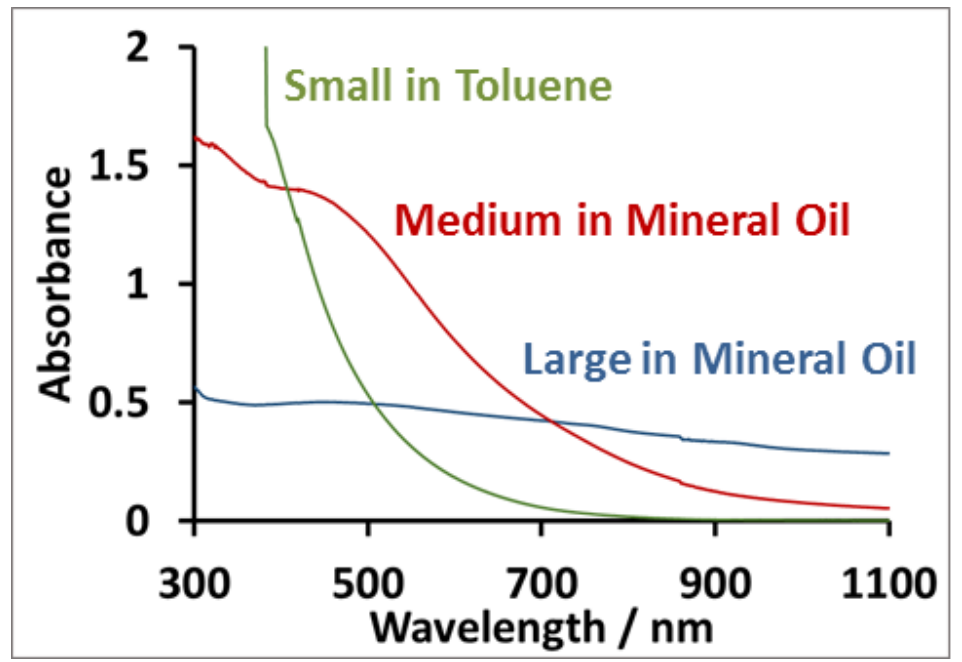

Figure S11: Absorption spectra derived from $-\log (R+T)$ referenced to the solvent of the SnS small nanoparticles (green), medium nanosheets (red), and large sheets (blue) in suspension at room temperature. 


\section{Figure S12}

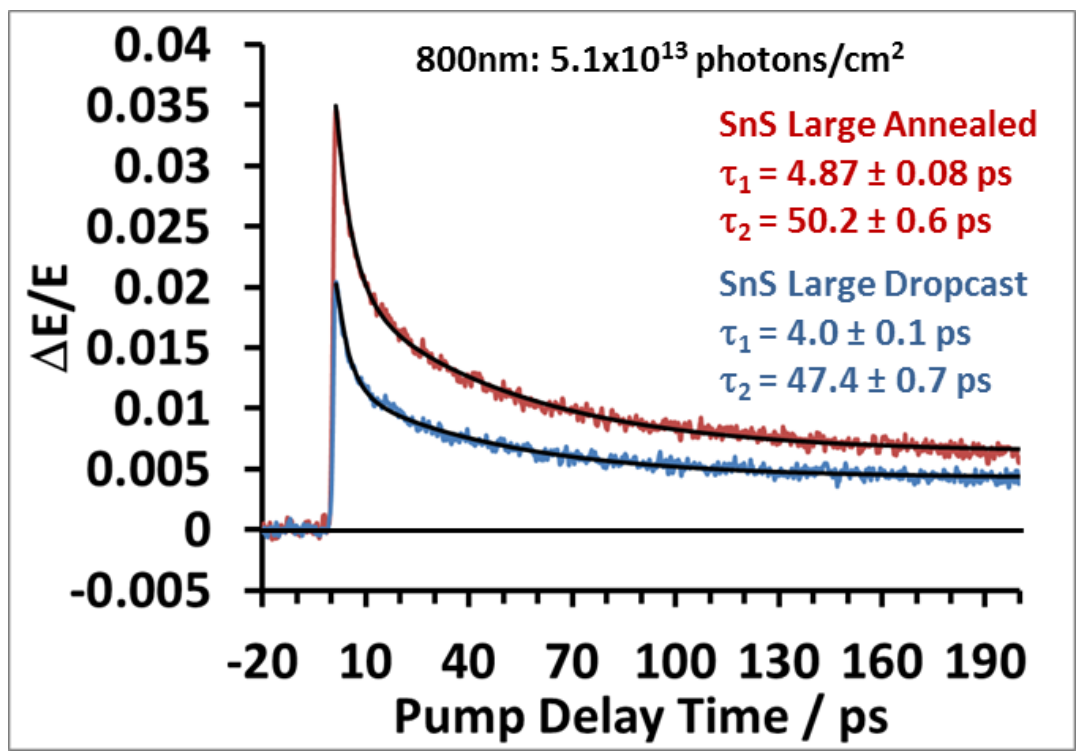

Figure S12: Comparison of the TRTS pump scan dynamics observed for the dropcast and annealed films of the SnS large sheets following $800 \mathrm{~nm}$ excitation at the indicated fluence. 